\title{
SALUD SEXUAL DE LOS MENORES Y JÓVENES DEL SISTEMA DE PROTECCIÓN Y EN CONFLICTO CON LA LEY
}

\author{
(1) Olga Fernández-García, (2) Beatriz Gil-Juliá, (1) María Dolores Gil-Llario \\ (1) Dpto de Psicología Evolutiva y de la Educación, Universitat de València, Valencia, \\ España \\ (2) Dpto de Personalidad, Evaluación y Tratamientos Psicológicos, Universitat de \\ València, Valencia, España \\ Olga.Fernandez-Garcia@uv.es
}

\begin{abstract}
Resumen
La literatura existente centrada en el estudio de la salud sexual de los/as niños/as y adolescentes, ha reportado resultados heterogéneos que varían en función de las experiencias vitales y de la historia sexual. Los/as niños/as y adolescentes del sistema de protección, a diferencia de los/as jóvenes en conflicto con la ley o de la población general, han sido retirados de sus hogares por sufrir abusos físicos o sexuales, entre otras razones. La vivencia de este tipo de sucesos en la infancia se ha vinculado con una serie de conductas disruptivas, entre las que destacan las conductas sexuales problemáticas. A pesar de ello, tanto los/as niños/as y adolescentes del sistema de protección como los/as jóvenes en conflicto con la ley, son poco accesibles y, en escasas ocasiones, se ha puesto el foco de atención en su salud sexual. Por este motivo, el grupo de investigación SALUSEX se ha embarcado en el presente proyecto que tiene como objetivo principal conocer la salud sexual de estos grupos poblacionales, mediante la realización de un estudio descriptivo que permita identificar posibles variables asociadas a un desarrollo sexual no saludable. La viabilidad de esta línea de investigación reside en dos convenios de colaboración con la DGIA y la Fundación Diagrama, respectivamente. Actualmente, se han evaluado mediante una batería de instrumentos a 326 niños/as y adolescentes (30.5\% mujeres; $69.5 \%$ hombres) de entre 11 y 19 años que residían en 48 hogares de acogimiento residencial situados en la provincia de Castellón y Valencia; y se ha comenzado a entrevistar a los/as jóvenes de centros socioeducativos. Como fin último, se busca que este estudio revierta en la sociedad mediante la elaboración de materiales específicos que permitan trabajar los posibles déficits de conocimientos y desarrollar estrategias útiles para atenuar las consecuencias que se derivan de un desarrollo sexual no saludable.
\end{abstract}

Palabras clave: Salud sexual; Niños/as; Adolescentes; Sistema de protección; Jóvenes en conflicto con la ley. 


\title{
SEXUAL HEALTH OF CHILDREN AND YOUNG PEOPLE IN THE CHILD WELFARE SYSTEM AND IN CONFLICT WITH THE LAW
}

\begin{abstract}
Existing literature focusing on the study of children's and adolescents' sexual health has reported heterogeneous results that vary according to life experiences and sexual history. Children and adolescents in the child welfare system, unlike young people in conflict with the law or the general population, have been removed from their homes for physical or sexual abuse, among other reasons. The experience of such events in childhood has been linked to a range of disruptive behaviours, including problematic sexual behaviour. Despite this, both children and adolescents in the child welfare system and young people in conflict with the law are hardly accessible and their sexual health has rarely been the center of attention. For this reason, the research group SALUSEX has embarked on this project whose main objective is to know the sexual health of these population groups, by conducting a descriptive study that allows to identify possible variables associated with sexual development not healthy. The viability of this line of research lies in two collaboration agreements with the DGIA and the Diagrama Foundation, respectively. Currently, 326 children and adolescents (30.5\% female; $69.5 \%$ male) between 11 and 19 years of age residing in 48 residential care homes in the provinces of Castellón and Valencia have been assessed using a battery of instruments; and interviews have begun with young people in socio-educational centres. Ultimately, this study is intended to have an impact on society through the development of specific materials to address possible knowledge deficits and to develop useful strategies to mitigate the consequences of unhealthy sexual development.
\end{abstract}

Keywords: Sexual health; Children; Adolescents; Child welfare system; Youth in conflict with the law. 


\section{Introducción}

La sexualidad es una dimensión intrínseca del ser humano y, por tanto, desde la infancia surgen los primeros comportamientos sexuales como parte del desarrollo normativo del menor (Larsson \& Svedin, 2002). Sin embargo, en contadas ocasiones la literatura científica se ha focalizado en el estudio de la salud sexual de los/as niños/as y adolescentes y, siempre que lo ha hecho, ha sido desde una perspectiva centrada en el análisis de los riesgos. A pesar de ello, los estudios existentes inciden en la heterogeneidad que caracteriza al desarrollo sexual infantil y adolescente al verse influenciado por las experiencias vitales y la historia sexual del individuo.

Los/as niños/as y adolescentes involucrados en el sistema de protección infantil han sido considerados tradicionalmente un grupo poblacional más vulnerable y con menor influencia social (Euser et al., 2014). En la mayoría de las ocasiones, han sufrido maltrato físico, emocional y/o sexual, se han criado en un ambiente de inestabilidad familiar en el que era común el abuso de sustancias por parte de los padres y la pobreza (Harden, 2004), y no han tenido una figura de referencia estable y accesible que actúe como fuente de apego (Tarren-Sweeney, 2008). Así, los múltiples factores de riesgo, sumados a la falta de factores de protección (relaciones familiares positivas, comunicación padre/madre-hijo, etc.), aumentan la probabilidad de presentar resultados adversos en múltiples dominios de la vida y de participar en conductas de riesgo para la salud (Harden, 2004). En un estudio con jóvenes extutelados, el 43\% informó haber estado embarazada o haber dejado embarazada a alguien antes de los 18 años (Collins et al., 2007), y, aunque en la mayoría de los casos se trató de embarazos no deseados, en algunas ocasiones reportaron que lo hicieron para sentirse queridas y atendidas, reescribir su infancia y dar el amor que no recibieron en su infancia a un/a niño/a (Aparicio et al., 2015). Así mismo, también son más propensos/as a experimentar un debut sexual temprano (Finigan-Carr et al., 2018), practicar sexo transaccional (Ahrens et al., 2012) y contraer ITS (Ahrens et al., 2010), como consecuencia, principalmente, de las vivencias de abuso/agresión sexual a las que han sido expuestos/as en su infancia. Cyr et al. (2012) que analizaron los comportamientos de 220 menores de 17 años de tres hogares de acogimiento residencial de Quebec reportaron una tasa de victimización sexual del 21\%, mientras Segura et al. (2015) encontraron que el 16.3\% de una muestra de jóvenes españoles del sistema de bienestar infantil habían denunciado abuso/agresión sexual por parte de un adulto conocido en el pasado. En relación con esto, Euser et al. (2013) señalaron que vivir en grandes grupos mixtos de niños/as que pueden haber sido abusados sexualmente y que exhiben comportamientos problemáticos, también podría contribuir al aumento del riesgo de sufrir victimización sexual. Así mismo, cabe resaltar que además de una historia de abuso sexual y físico (Merrick et al., 2008), la inestabilidad del lugar de residencia (Tarren-Sweeney, 2008) y la mayor exposición a comportamientos sexuales (desnudez familiar, limites deficientes, etc.) y a un patrón de socialización deficiente (Friedrich, 1997), también se ha relacionado con un comportamiento sexualizado atípico, ya que esto condiciona que presenten un déficit en el control inhibitorio y carezcan de mecanismos normativos de regulación del afecto (Lewis et al., 2007).

Algo semejante parece suceder con los/as adolescentes y jóvenes involucrados en el sistema de justicia juvenil debido, en parte, al vínculo que se ha encontrado entre la participación en este último y en el sistema de bienestar infantil (Oriol-Granado, et al., 2015; Pereda et al., 2017). Así, la victimización es una realidad también para este colectivo. Pereda et al. (2017), encontraron que el $96 \%$ de una muestra de 101 jóvenes 
involucrados en el sistema judicial español había sufrido algún delito contra su persona y el 95\% había sido expuesto a violencia comunitaria, cifras superiores a las encontradas en jóvenes de la misma edad de la población general (Pereda et al., 2014). Concretamente, preocupa el porcentaje de chicas delincuentes que reportan haber sufrido algún tipo de victimización sexual $(42.1 \%$ en Pereda et al., 2017 , y 36\% en Croysdale et al., 2008), ya que es sustancialmente más alto al encontrado en las muestras de la comunidad. En niños no se ha encontrado una relación tan estrecha entre la victimización sexual y la conducta delictiva, aunque sí han reportado altos niveles de violencia en las relaciones amorosas (Pereda et al., 2017). En este sentido, la alta exposición a situaciones de extrema violencia por parte de su círculo de amigos, vecinos y/o familiares (Ryan et al., 2013), como puede ser el asesinato de familiares o conocidos (Ford et al., 2008), las malas prácticas de crianza familiar (Fergusson et al., 2004), el nivel socioeconómico bajo (Murray y Farrington, 2010), y el contacto con sustancias psicoactivas y conductas sexuales de riesgo en el entorno más cercano, podrían colocar al individuo en un alto riesgo de sufrir polivictimización, lo que se relaciona con una mayor predisposición a presentar comportamientos antisociales (Mersky et al., 2012), graves problemas emocionales y conductas sexuales de riesgo (Sánchez et al., 2016).

A todo lo expuesto hasta el momento, cabe añadirle que, en escasas ocasiones, estos colectivos reportan haber recibido información sobre salud sexual y reproductiva (Melby et al., 2018), informando incluso de un menor acceso a estos servicios (Finigan-Carr et al., 2018). Esto podría ser explicado por los problemas para comunicarse con los médicos que suelen presentar estas jóvenes y el deficiente compromiso que establecen con la escuela, fuente fundamental de información afectivo-sexual (Hudson, 2012). Además, en aquellas ocasiones que recibieron educación sexual ésta llegó tarde, ya que la edad de las primeras experiencias sexuales es anterior al promedio (Boustani et al., 2015).

Teniendo en cuenta todo lo comentado, y que ambos colectivos son poco accesibles, por lo que en escasas ocasiones se ha puesto el foco de atención en su salud sexual, el grupo de investigación SALUSEX se propone conocer la salud sexual de estos grupos poblacionales mediante la realización de un estudio descriptivo que permita identificar posibles variables asociadas a un desarrollo sexual no saludable. Así, el presente trabajo tiene el cometido de presentar esta línea de investigación que ocupa actualmente al grupo de investigación SALUSEX y mostrar los pasos realizados.

\section{Método}

\section{Participantes}

La población diana de la presente línea de investigación son tanto los/as niños/as y adolescentes involucrados en el sistema de protección infantil como los/as jóvenes en conflicto con la ley, que residan en centros de acogimiento residencial $o$ socioeducativos, respectivamente, ubicados en la Comunitat Valenciana.

Respecto al primer colectivo, los/as niños/as y adolescentes del sistema de protección son personas menores de edad cuya guarda o tutela pertenece a la administración pública. Así, los/as participantes tienen entre 11 y 19 años, ya que, aunque con la mayoría de edad dejan los recursos de acogimiento, también se va a evaluar a aquellos jóvenes que conviven en hogares de emancipación. Así, los/as 
participantes residen en hogares o residencias de acogimiento residencial general, específicos de problemas graves de conducta, de emancipación o de proyecto migratorio (para menores migrantes sin referentes familiares) de Castellón y Valencia.

Respecto a los/as jóvenes inmersos en el sistema judicial juvenil, se trata de personas menores de edad respecto a quienes se ha adoptado una medida judicial como consecuencia de haber cometido un hecho constitutivo de infracción penal, aun cuando en el momento de ejecución de la medida hubieran alcanzado la mayoría de edad. Así, todos los participantes están cumpliendo una medida judicial de medio cerrado en una residencia socioeducativa de la Comunitat Valenciana.

Como criterio de inclusión, todos los participantes deben presentar un buen nivel de español y un nivel de comprensión que permita responder adecuadamente a las preguntas planteadas. En cualquier caso, se asegura la adaptación de las cuestiones a las características y conocimientos de la persona evaluada, preservando siempre el contenido principal de la pregunta.

\section{Instrumentos}

Para la consecución del objetivo principal de esta línea de investigación, se ha diseñado un estudio cuantitativo que nos va a permitir obtener información objetiva y de forma exhaustiva a través de la implementación de instrumentos de evaluación validados, en formato de entrevista semiestructurada. Se busca recoger información sobre las siguientes variables:

Información Sociodemográfica. Cuestionario ad-hoc que recoge información sobre variables sociodemográficas (género, edad, nacionalidad, problemas de salud/salud mental, situación escolar/laboral, historia en el sistema de protección, historial de visitas e información de la familia biológica) a través de una serie de preguntas con distinto formato de respuesta (dicotómico, elección múltiple, abierta, etc.). En el caso de los/as jóvenes en conflicto con la ley, también se incluyen preguntas relativas a su historial delictivo. Se trata del único cuestionario cumplimentado por los profesionales del recurso basándose en los informes existentes del/de la niño/a o adolescente.

Conocimientos y actitudes sexuales. A través de un cuestionario compuesto por 59 ítems tipo Likert (verdadero, falso, no seguro/a) se evalúan los conocimientos y actitudes hacia diferentes aspectos de la sexualidad en un sentido amplio. Se emplea otro cuestionario de 20 reactivos con 6 opciones de respuesta para evaluar las actitudes ambivalentes hacia las mujeres.

Búsqueda de sensaciones sexuales, adicción al sexo y consumo de cibersexo. Se emplea un instrumento de 11 ítems tipo Likert que recogen el grado de acuerdo/desacuerdo con una serie de declaraciones relacionadas con la búsqueda de sensaciones en el ámbito de la sexualidad; otra escala de 25 ítems dicotómicos que evalúan los principales ejes del diagnóstico de la adicción al sexo; $\mathrm{y}$, un último instrumento de 24 ítems dicotómicos que permite evaluar los comportamientos asociados al uso de internet con fines sexuales.

Asertividad sexual, conductas sexuales de riesgo e impulsividad. Para la evaluación de estos aspectos, en primer lugar, se elaboró un instrumento ad-hoc de 24 ítems con 4 opciones de respuesta para evaluar las habilidades asertivas en las relaciones sexuales; 
otro instrumento de 8 ítems para evaluar las conductas de sexting y las motivaciones que han llevado a la persona a practicarlo; y, otro instrumento de 15 ítems con 4 opciones de respuesta que evalúa la impulsividad en diferentes ámbitos de su vida.

Bienestar psicológico, regulación emocional, autoestima y empatía. El instrumento empleado para evaluar el bienestar psicológico está formado por 20 ítems tipo Likert que miden la autoaceptación, las relaciones interpersonales positivas, la autonomía, y el desarrollo vital. En el caso de la regulación emocional, el instrumento tiene 36 ítems tipo Likert que indagan sobre la falta de conciencia, claridad y respuestas emocionales, las dificultades para controlar impulsos y para persistir en una tarea con malestar, y el acceso limitado a estrategias de regulación emocional. Así mismo, dos cuestionarios de 10 y 9 ítems permiten explorar los sentimientos de valía personas y respeto hacia uno mismo, y la empatía afectiva y emocional, respectivamente.

Vínculo de apego e historia de maltrato y abuso sexual. Se emplea un cuestionario de 32 ítems para medir las representaciones del apego, y otros dos instrumentos de $17 \mathrm{y}$ 6 ítems para evaluar la frecuencia y alcance de varias experiencias de victimización infantil y adolescente.

\section{Procedimiento}

La viabilidad de la presente línea de investigación pasa por la elaboración de dos convenios de colaboración, uno junto a la Dirección General de Infancia y Adolescencia (DGIA) perteneciente a la Conselleria de Igualdad y Políticas Inclusivas (CIPI), órgano competente para ejecutar las medidas de protección de menores en la Comunitat Valenciana, y otro junto a la Fundación Diagrama, entidad encargada de la gestión de recursos de acogimiento residencial y socioeducativos.

En primer lugar, se realizó una extensa búsqueda bibliográfica que nos permitió, no solo obtener información acerca de la salud sexual de los/as niños/as, adolescentes y jóvenes y de las características de los colectivos poblaciones estudiados, sino también identificar instrumentos de evaluación validados que nos permitieran indagar sobre aspectos clave en el desarrollo afectivo-sexual infantil y adolescente. Seguidamente, se presentó el proyecto a la Comisión de Ética de la Universitat de Valencia y se obtuvo su informe favorable, lo que permitió comenzar el contacto con los/as directores/as de los hogares y residencias de acogimiento residencial y socioeducativas, a quienes se les expuso el proyecto y se les explicó la forma de proceder con las evaluaciones. Así mismo, previamente al comienzo de éstas, se formó a las personas del equipo de investigación SALUSEX que iban a participar en la evaluación de los participantes, con el objetivo de que los datos recogidos no se vieran sesgados por la subjetividad del evaluador.

\section{Resultados}

\section{Datos recogidos hasta el momento}

Desde que dio comienzo esta fase del proyecto en enero del 2020, se ha evaluado a un total de 326 niños/as y adolescentes involucrados en el sistema de protección infantil de la Comunitat Valenciana. Para ello se acudió a un total de 48 residencias/hogares de 
las cuales 13 estaban ubicadas en la provincia de Castellón y 35 en la provincia de Valencia. A continuación, en la tabla 1, se recoge de forma detallada la tipología de las residencias/hogares a los que se ha acudido:

Tabla 1 Tipología de las residencias/hogares a los que hemos acudido

\begin{tabular}{lcc}
\cline { 2 - 3 } Tipología de la residencia/hogar & \multicolumn{2}{c}{ Provincia } \\
\hline De acogimiento general & 11 & Valencia \\
Específicos para problemas de conducta & - & 3 \\
De emancipación & 2 & 6 \\
De proyecto migratorio & - & 4 \\
\hline
\end{tabular}

Por lo que respecta a las características de los participantes (ver tabla 2), el 68.3\% son hombres frente al $31.7 \%$ que son mujeres, debido a la proporción más alta de personas menores de edad del sexo masculino que viven en recursos de acogimiento residencial. En lo relativo a la edad, los participantes tienen entre 11 y 19 años $(\mathrm{M}=15.77, \mathrm{DE}=1.598)$, y el $12.4 \%$ presenta diversidad funcional física y/o intelectual. Así mismo, el 32.1\% de los participantes son migrantes sin referentes familiares.

\begin{tabular}{|c|c|c|}
\hline & $\mathrm{N}$ & $\%$ \\
\hline \multicolumn{3}{|l|}{ Género } \\
\hline Hombres & 170 & 68.3 \\
\hline Mujeres & 79 & 31.7 \\
\hline \multicolumn{3}{|l|}{ Edad } \\
\hline 11 años & 1 & 0.4 \\
\hline 12 años & 9 & 3.6 \\
\hline 13 años & 14 & 5.6 \\
\hline 14 años & 27 & 10.9 \\
\hline 15 años & 49 & 19.8 \\
\hline 16 años & 47 & 19 \\
\hline 17 años & 79 & 31.9 \\
\hline 18 años & 17 & 6.9 \\
\hline 19 años & 5 & 2 \\
\hline \multicolumn{3}{|c|}{ Diversidad funcional } \\
\hline Sí & 31 & 12.4 \\
\hline No & 218 & 87.6 \\
\hline
\end{tabular}

En el caso de las residencias socioeducativas, las evaluaciones se encuentran en una fase más inicial.

\section{Procedimiento de evaluación empleado}

Para la recopilación de los datos se han realizado entrevistas semiestructuradas e individualizadas dirigidas por los profesionales del equipo de investigación SALUSEX que habían sido formados previamente para ello. Se procuró que se llevaran a cabo en una estancia privada de la residencia/hogar donde residía el/la niño/a o adolescentes, en la que únicamente estuvieran presentes el/la participante y el/la entrevistador/a (ningún 
profesional de recurso), con el objetivo de crear un ambiente lo suficientemente íntimo como para que el/la evaluado/a se sintiera a gusto para abordar esos asuntos. Previamente al inicio de ésta, se le informaba al/a la participante del objetivo y las condiciones del estudio, aunque ya lo hubieran hecho desde el recurso, y se destinaban unos minutos a establecer buen rapport con él/ella. Se dejaba clara la voluntariedad de su participación en el estudio, la posibilidad de abandonar en cualquier momento, y la confidencialidad de los datos recopilados, siendo nula la información identificativa recogida. También se les informaba de la duración aproximada de las entrevistas (1:30h aprox.), siendo preferible que se completara en un único momento temporal. Así mismo, el/la entrevistador/a podía dar la oportunidad de que la batería de instrumentos fuera cumplimentada de forma autónoma por parte del/de la participante, si reunía las condiciones necesarias de comprensión y dominio del idioma como para responder adecuadamente a las preguntas. Sin embargo, en contadas ocasiones el/la participante optó por cumplimentarlo como autoinforme. Huelga decir que las entrevistas fueron realizadas adaptándonos no solo a la organización del propio recurso (actividades establecidas, disponibilidad de profesionales, etc.), sino también a la disponibilidad y horario del/de la participante.

Así mismo, la implementación de la batería de instrumentos siguió un orden cuidadosamente establecido (mostrado en el apartado "instrumentos") que permitía pasar del abordaje de aspectos menos invasivos a aquellos más invasivos. Sin embargo, este podía verse alterado siempre que hubiera una causa de peso, como podía ser la incomodidad o el exceso de emotividad del/de la participante frente a ciertos temas.

Por último, cabe añadir que, en ocasiones determinadas, se contó con la colaboración de mediadores interculturales para la traducción de términos muy concretos al árabe.

\section{Conclusiones}

Los comportamientos sexualizados, como mecanismo de afrontamiento inapropiado, no solo pueden ser un indicio de desregulación afectiva y actuar como precursores de otros comportamientos problemáticos (p. ej. delincuencia juvenil), sino que pueden estar encubriendo abusos sexuales no reportados hasta el momento. De aquí la importancia de prestar la atención necesaria a esta área del desarrollo humano y no centrarse únicamente en las formas más visibles de victimización sobre las que se ha basado la decisión de instaurar diligencias asistenciales. Además, como señaló la Association for Treatment of Sexual Abusers (2014), los problemas de conducta, incluidos los problemas de conducta sexual, son las principales razones por las que fallan los acogimientos familiares, lo que, al mismo tiempo, provoca muchos problemas emocionales y conductuales. Por todo ello, la identificación de las variables predictoras de un desarrollo sexual no saludable, objetivo principal de esta línea de investigación, va a ser esencial.

En este sentido, el propósito principal de este proyecto es que el estudio de la salud sexual de los/as niños/as y adolescentes del sistema de protección y de los/as jóvenes en conflicto con la ley revierta en la sociedad. Para ello, este estudio puede ayudar a la construcción de materiales específicos de intervención que permitan trabajar los déficits en conocimientos, habilidades y actitudes sexuales detectados, así como contribuir al desarrollo de estrategias útiles para atenuar las consecuencias que se derivan de un 
desarrollo sexual no saludable. Concretamente, esto pasa por la creación de programas de prevención e intervención orientados a atenuar las conductas sexuales de riesgo más frecuentes en este colectivo, evitando que lleguen a gestarse actitudes negativas como el sexismo o la homofobia, y se desarrollen comportamientos que puedan ser dañinos para uno mismo y para la sociedad, como la violencia de género. En este sentido, proveer a los profesionales que diariamente están con estos colectivos de estrategias claras sobre cómo abordar temas de salud sexual y reproductiva, también va a ser una labor esencia a la hora de asegurar intervenciones más sensibles y efectivas.

Por último, teniendo en cuenta que los/as niños/as y adolescentes del sistema de protección y los/as jóvenes en conflicto con la ley han sido tradicionalmente desatendidos y negados como parte de la sociedad, esta línea de investigación se propone también darles visibilidad y mostrar una realidad para muchos inexistente.

\section{Referencias}

Ahrens, K.R., Katon, W., McCarty, C., Richardson, L.P., y Courtney, M. (2012). Association between childhood sexual abuse and transactional sex in youth aging out of foster care. Child Abuse \& Neglect, 36, 75-80. https://doi.org/10.1016/j.chiabu2011.07.009.

Ahrens, K.R., Richardson, L.P., Courtney, M.E., McCarty, C., Simoni, J., y Katon, W. (2010). Laboratory-diagnosed sexually transmitted infections in former foster youth compared with peers. Pediatrics, 126(1), 97-103. https://doi.org/10.1542/peds.2009-2424

Aparicio, E., Pecukonis, E.V., y O'Neale, S. (2015). "The love that I was missing": Exploring the lived experience of motherhood among teen mothers in foster care. Child Youth Services Review, 51, 44-54. https://doi.org/10.1016/i.childyouth.2015.02.002.

Association for Treatment of Sexual Abusers. (2014). Fact sheet. Children with sexual behavior problems. Recuperado

de http://www.atsa.com/children-sexual-behavior-problems

Boustani, M.M., Frazier, S.L., Hartley, C., Meinzer, M., y Hedemann, E.R. (2015). Perceived benefits and proposed solutions for teen pregnancy: Qualitative interviews with youth care workers. American Journal of Orthopsychiatry, 85(1), 80-92. https://doi.org/10.1037/ort0000040

Collins, M.E., Clay, C.M., y Ward, R. (2007). Leaving care in Massachusetts: Policy and supports to facilitate transition to adulthood. Boston University School of Social Work.

Croysdale, A.E., Drerup, L.C., Bewsey, K., y Hoffmann, N.G. (2008). Correlates of victimization in a juvenile justice population. Journal of Aggression, Maltreatment \& Trauma, 17, 103-117. https://doi.org/10.1080/10926770802250876

Cyr, K., Chamberland, C., Lessard, G., Clément, M.E., Wemmers, J.A., Collin-Vézina, D., Gagné, M.H., y Damant, D. (2012). Polyvictimization in a child welfare sample 
of children and youths. Psychology of Violence, 2(4), 385-400. https://doi.org/10.1037/a0028040

Euser, S., Alink, L.R.A., Tharner, A., Van Ijzendoorn, M.H., y Bakermans-Kranenburg, M.J. (2014). Out of home placement to promote safety? The prevalence of physical abuse in residential and foster care. Child Youth Service Review, 37, 64-70. https://doi.org/10.1016/i.childyouth.2013.12.002.

Euser, S., Alink, L.R.A., Tharner, A., Van Ijzendoorn, M.H., y Bakermans-Kranenburg, M.J. (2013). The prevalence of child sexual abuse in out-of-home care: A comparison between abuse in residential and in foster care. Child Maltreatment, 18(4), 221-231. https://doi.org/10.1177/1077559513489848.

Fergusson, D.M., Swain-Campbell, N.R., y Horwood, L.J. (2004). How does childhood economic disadvantage lead to crime? Journal of Child Psychology and Psychiatry, 45, 956-966. https://doi.org/10.1111/j.1469-7610.2004.t01-1-00288.x

Finigan-Carr, N., Steward, R., y Watson, C. (2018). Foster youth need sex ed, too!: Addressing the sexual risk behaviors of system-involved youth. American Journal of Sexuality Education, 13(3), 310-323. https://doi.org/10.1080/15546128.2018.1456385.

Ford, J.D., Hartman, J.K., Hawke, J., y Chapman, J.C. (2008). Traumatic victimization, posttraumatic stress disorder, suicidal ideation, and substance abuse risk among juvenile justice-involved youths. Journal of Child \& Adolescent Trauma, 1, 75-92. https://doi.org/10.1080/19361520801934456

Friedrich, W.N. (1997). Child Sexual Behavior Inventory professional manual. Psychological Assessment Resources.

Harden B. (2004). Safety and stability for foster children. The Future of Children, 14(1), 30-47. https://doi.org/10.2307/1602753

Hudson, A.L. (2012). Where do youth in foster care receive information about preventing unplanned pregnancy and sexually transmitted infections? Journal of Pediatric Nursing, 27(5), 443-450. https://doi.org/10.1016/j.pedn.2011.06.003

Larsson, I., y Svedin, C.G. (2002). Sexual experiences in childhood: Young adults' recollections. Archives of Sexual Behavior, 31(3), 263-273. https://doi.org/10.1023/a:1015252903931

Lewis, E.E., Dozier, M., Ackerman, J., y Sepulveda-Kozakowski, S. (2007). The effect of placement instability on adopted children's inhibitory control abilities and oppositional behavior. Developmental Psychology, 43(6), 1415-1427. https://doi.org/10.1037=0012-1649.43.6.1415

Melby, J., Rouse, H., Jordan, T., y Weems, C. (2018). Pregnancy and Parenting among Iowa Youth Transitioning from Foster Care: Survey and Focus Group Results. Iowa State University.

Merrick, M.T., Litrownik, A.J., Everson, M.D., y Cox, C.E. (2008). Beyond Sexual Abuse: The Impact of Other Maltreatment Experiences On Sexualized Behaviors. Child Maltreatment, 13(2), 122-132. https://doi.org/10.1177/1077559507306715 
Mersky, J.P., Topitzes, J., y Reynolds, A.J. (2012). Unsafe at any age: Linking childhood and adolescent maltreatment to delinquency and crime. Journal of Research in Crime and Delinquency, 49, 295-318. https://doi.org/10.1177/0022427811415284

Murray, J., y Farrington, D.P. (2010). Risk factors for conduct disorder and delinquency: Key findings from longitudinal studies. Canadian Journal of Psychiatry, 55, 633-642. https://doi.org/10.1177/070674371005501003

Oriol-Granado, X., Sala-Roca, J., y Filella-Guiu, G. (2015). Juvenile delinquency in youths from residential care. European Journal of Social Work, 18, 211-227. https://doi.org/10.1080/13691457.2014.892475

Pereda, N., Abad, J., y Guilera G. (2017). Victimization and Polyvictimization of Spanish Youth Involved in Juvenile Justice. Journal of Interpersonal Violence, 32(21), 3272-3300. https://doi.org/10.1177/0886260515597440

Pereda, N., Guilera, G., y Abad, J. (2014). Victimization and polyvictimization of Spanish children and youth: Results from a community sample. Child Abuse \& Neglect, 38, 640-649. https://doi.org/10.1016/j.chiabu.2014.01.019

Ryan, J.P., Williams, A.B., y Courtney, M.E. (2013). Adolescent neglect, juvenile delinquency and the risk of recidivism. Journal of Youth and Adolescence, 42(3), 454-465. https://doi.org/10.1007/s10964-013-9906-8.

Sánchez, R., Enríquez, D., y Robles, S. (2016). Evaluación de un programa de intervención para promover la salud sexual en adolescentes mexicanos en conflicto con la ley. Acta Colombiana de Psicología, 19(2), 257-268. https://www.redalyc.org/articulo.oa?id=79847072011

Segura, A., Pereda, N., Abad, G., y Guilera, G. (2015). Victimization and polyvictimization among Spanish youth protected by the child welfare system. Children and Youth Services Review, 59, 105-112. https://doi.org/10.1016/j.childyouth.2015.10.011

Tarren-Sweeney, M. (2008). Predictors of problematic sexual behavior among children with complex maltreatment histories. Child Maltreatment, 13(2), 182-198. $\underline{\text { https://doi.org/10.1177=1077559508316043 }}$ 